\title{
Transformación, categorización y reconocimiento del conflicto armado colombiano $^{1}$
}

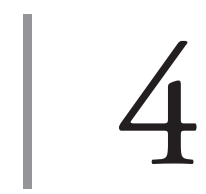

https://doi.org/10.21830/9789585287860.04

\author{
Erika Ramirez Benitez ${ }^{2}$ \\ Henry Mauricio Acosta Guzmán ${ }^{3}$ \\ Escuela Superior de Guerra "General Rafael Reyes Prieto" \\ Jaime Cubides-Cárdenas ${ }^{4}$ \\ Escuela Militar de Cadetes "General José María Córdova”
}

\section{Resumen}

El capítulo analiza las complejas transformaciones que ha tenido el conflicto armado colombiano, los actores y los factores que han permitido su prolongación como el más antiguo de Latinoamérica. Siguiendo un enfoque cualitativo, con un método descriptivo desarrollado por medio de herramientas como el análisis documental y de información, se estudian las distintas categorizaciones que los gobiernos colombianos han hecho del conflicto armado, así como la normativa que se ha incorporado

1 Este capítulo expone en forma conjunta los resultados de tres proyectos de investigación. Proyecto "El conflicto: experiencias y aprendizajes internacionales. Una reflexión desde los procesos de paz" del grupo de investigación de Ciencias Militares de la Escuela Militar de Cadetes "General José María Córdova”, categorizado en B por Minciencias y con código de registro COL0082556. Proyecto "Nuevas amenazas en el siglo XXI, fronteras y Derechos Humanos - Fase II" del grupo de investigación Centro de Gravedad de la Escuela Superior de Guerra "General Rafael Reyes Prieto", categorizado en A1 por Minciencias y con código de registro COL0104976. Proyecto "Ética, liderazgo y familia en las Fuerzas Militares como factores para la construcción de Estado" del grupo de investigación Masa Crítica de la Escuela Superior de Guerra "General Rafael Reyes Prieto", categorizado en B por Minciencias y con código de registro COL0123247. Los puntos de vista y los resultados de este capítulo pertenecen a los autores y no reflejan necesariamente los de las instituciones participantes.

2 Politóloga de la Universidad Nacional de Colombia. Magíster en Estrategia y Geopolítica de la Escuela Superior de Guerra "General Rafael Reyes Prieto”, Bogotá, D. C., Colombia. Estudiante de maestría en Análisis de Problemas Políticos, Económicos e Internacionales Contemporáneos de la Universidad Externado de Colombia y la Academia Diplomática Augusto Ramírez Ocampo de Colombia. Investigadora junior reconocida por Minciencias. Asesora en investigación de la Vicedirección de Investigación de la Escuela Superior de Guerra “General Rafael Reyes Prieto”, Bogotá, D. C, Colombia. ORCID: https://orcid.org/0000-0001-9830-8457

3 Politólogo de la Universidad Nacional de Colombia. Magíster en Seguridad y Defensa Nacionales de la Escuela Superior de Guerra "General Rafael Reyes Prieto”, Bogotá, D. C., Colombia. Joven investigador del Departamento de Ética y Liderazgo de la Escuela Superior de Guerra, Colombia. ORCID: https://orcid.org/00000003-4485-8845

4 Abogado y especialista en Derecho Público de la Universidad Autónoma de Colombia. Especialista y magíster en Docencia e Investigación con énfasis en las Ciencias Jurídicas de la Universidad Sergio Arboleda, y magíster en Derecho de la misma casa de estudios. Investigador senior reconocido y categorizado por Minciencias. Docente investigador de la Facultad de Relaciones Internacionales de la Escuela Militar de Cadetes "General José María Córdova”, Bogotá, D. C., Colombia. ORCID: https://orcid.org/0000-0002-6542-6892. Contacto: jaime. cubides@esmic.edu.co 
relacionada con el Derecho Internacional Humanitario desde la adopción del Protocolo II Adicional a los Convenios de Ginebra del año 1949. Así, este trabajo cumple tres objetivos: (1) caracterizar la manera en que se ha entendido el conflicto armado en Colombia, (2) identificar cómo los gobiernos colombianos han interpretado el conflicto armado para la organización de la Estrategia Nacional y (3) determinar la perspectiva actual respecto al conflicto armado. La investigación concluye que, por una parte, el Estado ha tenido una evolución sistemática en la forma como hace frente a las amenazas tradicionales en el marco de la guerra irregular, por ejemplo, las guerrillas revolucionarias. Por otra parte, conforme a los estándares internacionales y dadas las situaciones derivadas del conflicto y relacionadas con las organizaciones criminales, el Estado sigue haciendo uso de marcos normativos referentes al Derecho Internacional Humanitario, pese a que en algunas situaciones se ha impedido establecer una estrategia nacional contundente para confrontar a los actores armados ilegales.

Palabras clave: Estado; estrategia nacional; conflicto armado; gobierno nacional.

\section{Introducción}

El desarrollo propio del Estado colombiano y, sobre todo, los elementos en su construcción han tenido temporalidades específicas, en las cuales se han expresado diversas lógicas de violencias en momentos históricos determinados. De este modo, como expone Cárdenas (2013), "desde el mismo origen del Estado colombiano, la violencia y los conflictos han sido un elemento constitutivo de la identidad nacional y la construcción estatal" (p. 42). Uno de estos momentos, por ejemplo, fue El Bogotazo, un hito histórico que aconteció el 9 de abril de 1948 en la ciudad de Bogotá a partir del asesinato del líder liberal Jorge Eliécer Gaitán. Este suceso marcaría un punto determinante para el desarrollo posterior de "La Violencia", por cuanto antecedió el surgimiento y proliferación de violencias en el Estado colombiano que perduran hasta la fecha.

Esto se puede relacionar con lo que plantea el Barcelona Centre for International Affairs (CIDOB, s. f.):

Colombia se ha caracterizado por una brecha social entre una élite política con una fuerte presencia regional [...], por otro lado, grupos minoritarios como los indígenas, afrodescendientes y campesinos que no han contado con el mismo acceso a los derechos de propiedad ni a los servicios del Estado. (párr. 6)

5 Periodo histórico en el cual se desarrollaron acciones de violencia a partir de etapas como el bipartidismo, en donde las diferencias entre el Partido Liberal y el Conservador alcanzaron un punto crítico. Estas tensiones se empezaron a manifestar en concepciones de predominio y aversión, que desencadenaron efectos como muerte, exclusión, entre otros factores (Rehm, 2014). Asimismo, también es pertinente enunciar que, en el marco del desarrollo de este suceso, las minorías que se ubicaban por fuera de los ideales de estos dos partidos políticos también estaban al margen de poder participar políticamente, por ello también se generaron acciones violentas en contra de estos. 
Junto con esta situación, son varias las dinámicas que han posibilitado que el conflicto armado interno se haya desarrollado durante aproximadamente sesenta años, en los cuales factores como la desigualdad presente en la repartición de la tierra, la precaria participación política de las minorías y distintos fenómenos que fueron emergiendo como el narcotráfico, la convergencia entre lo criminal, lo insurgente y lo terrorista, desataron acciones de violencia en el territorio colombiano. En el marco de esta dinámica, tuvieron lugar diversos sucesos como la aparición de las insurgencias, las cuales mantenían una motivación ideológica que desarrollaron en mayor medida por medio de las armas y aludiendo principalmente a una proximidad a la izquierda colombiana. Este es el caso de las Fuerzas Armadas Revolucionarias de Colombia - Ejército del Pueblo (FARC-EP), el Ejército de Liberación Nacional (ELN), el Ejército Popular de Liberación (EPL), el Movimiento 19 de Abril (M-19), entre otros (CIDOB, s.f.).

Posteriormente, surgieron grupos paramilitares como las Autodefensas Unidas de Colombia (AUC), cuya tendencia a contrarrestar a las guerrillas recrudeció en mayor magnitud las acciones violentas. Esta situación se agravó en 1980 por la generación del narcotráfico (CIDOB, s. f.), que concentraba en el país principalmente el primer eslabón de la cadena: la producción, sin dejar de lado que la comercialización y el consumo también estaban presentes.

En este escenario, la dinámica propia de la confrontación armada tuvo una serie de cambios debido a la multiplicidad de actores, de causas, de coyunturas y acciones que se desarrollaban, así como a los efectos que traía consigo la generación de esta situación que perdura hasta la actualidad. En este contexto, el Estado colombiano, en cabeza del gobierno nacional, como conductor político, ha tomado una posición específica respecto a la definición de la situación de violencias que acontecía en el territorio nacional. A partir de lo expresado surge la siguiente pregunta de investigación: ¿De qué manera ha sido el proceso de desarrollo del conflicto armado en Colombia teniendo en cuenta la transformación, categorización y reconocimiento que ha tenido?

Teniendo en cuenta lo anterior, la perspectiva temporal de este trabajo parte del año 1991 como el escenario en donde se presentó un hito en la historia colombiana: la consolidación de la Constitución Política de Colombia como resultado de un proceso de participación de los sectores políticos que estuvo antecedido por la realización de la Asamblea Nacional Constituyente. A partir de la Carta Política se trazaron nuevos lineamientos para organizar el Estado que dieron mayor relevancia a la democracia y la participación.

Específicamente, este trabajo analiza el desarrollo del conflicto armado en Colombia teniendo en cuenta la transformación, categorización y reconocimiento 
que ha tenido. Con este propósito se han planteado tres objetivos: (1) caracterizar la manera en que se ha entendido el conflicto armado en Colombia, (2) identificar cómo los gobiernos colombianos han interpretado el conflicto armado para organizar la Estrategia Nacional y (3) determinar la perspectiva actual respecto al conflicto armado. Finalmente, se establecen las conclusiones del estudio.

\section{Caracterización del conflicto armado colombiano}

El Derecho Internacional Humanitario (DIH) ${ }^{6}$, entendido como las normas de alcance internacional por medio de las cuales se "trata de limitar los efectos de los conflictos armados, proteger a las personas que no participan o que ya no participan en los combates y limitar los medios y métodos de hacer la guerra" (Comité Internacional de la Cruz Roja [CICR], 2004), permite categorizar los conflictos armados dependiendo de su alcance e intensidad.

Según la tipología del DIH, se podría reconocer el caso colombiano como un conflicto de carácter no internacional. Asimismo, se debe tener en cuenta que en el año 1996 entró en vigor el Protocolo II Adicional a los Convenios de Ginebra (1949), el cual fue creado en el marco del fin de la Segunda Guerra Mundial, en donde se dieron varios conflictos de carácter interno que requerían regulación. De este modo, se normatizan las regulaciones sobre aspectos generales de los conflictos armados no internacionales o internos respecto a las garantías a la protección de la población civil y quienes no se encuentran inmersos en este tipo de confrontaciones, además se toma en cuenta el principio de la humanidad como eje. Este protocolo, según el CICR (2016) aplica en

conflictos no internacionales entre las fuerzas armadas de un país y fuerzas disidentes o grupos armados organizados, así como entre grupos armados dentro del mismo país. Esto incluye a los grupos que controlen una parte del territorio desde el cual puedan realizar operaciones armadas sostenidas.

En este sentido, junto con el artículo tercero común a los Convenios de Ginebra que determina la regulación, son los dos elementos que permiten aproximarse a una delimitación de los conflictos armados no internacionales. Por lo tanto,

6 "Este sistema universal de protección de los Derechos Humanos permitió la consagración de instrumentos internacionales en derechos específicos para el tratamiento, atención y protección de aquellas personas que por los conflictos se convirtieron en víctimas. Así, nace como obligación internacional a cargo del Estado, la protección de los derechos en medio de un contexto de conflicto - ya sea interno o internacional—, en los términos de los primeros artículos comunes de los cuatro convenios de Ginebra y sus protocolos adicionales" (Vallejo, Ramírez \& Cubides-Cárdenas, 2017, p. 56). 
los aspectos derivados de las normas generales de Ginebra aplican principalmente para quienes inciden directamente en el desarrollo de la confrontación, es decir, las partes que se enfrentan en el interior del Estado.

Teniendo en cuenta el marco explicativo, la particularidad de este fenómeno violento, como lo denomina Trejos (2013), es la amplitud, ya que su desarrollo ha involucrado múltiples ámbitos, desde políticos, sociales, económicos, culturales, militares hasta históricos, lo cual hace que su abordaje también esté lleno de dificultades. De allí que las medidas y acciones realizadas para solucionarlo no fueran claras y, por ende, no generaran los resultados esperados hasta que se desarrolló el proceso de paz actual, que puede considerarse como un hito histórico que generó una serie de reconfiguraciones en la esfera estatal, propias de los procesos de paz.

En Colombia, desde la década de los ańos sesenta se fueron particularizando una serie de actividades que emergieron por la dinámica propia del conflicto y por los actores partícipes de este periodo complejo, catastrófico y de incertidumbre. En estas actividades convergieron diversos fenómenos de violencia que se integraron progresivamente a las acciones ilegales — como lo fue el narcotráfico-, que desarrollaron aspectos ligados a la profundización de las violencias y, con ello, la afectación de los Derechos Humanos por medio de crímenes como secuestros, homicidios, atentados, entre otros (Garay \& Ramírez-Benítez, 2017, p. 421).

Poco a poco y a medida que transcurría el conflicto se hizo necesario transformar los medios y modos de la estrategia nacional, lo cual dependía de las prioridades que el gobierno determinara y teniendo presente siempre la protección del interés nacional (supervivencia del Estado, bienestar económico, sistema de creencias y valores), así como la garantía de los fines constitucionales del Estado colombiano. En palabras de Corbacho (2011), en este nivel estratégico los objetivos nacionales, ya sea en tiempos de guerra o de paz, deben estar orientados al restablecimiento progresivo y continuo de la política, "lo que implica la irrupción más corta y menos costosa de la vida normal del país” (p. 19).

En este contexto, resulta importante conocer los principales elementos que permiten comprender la trascendencia de este fenómeno histórico que aún es vigente. En este sentido, se encuentra que el desarrollo del conflicto armado interno en Colombia tiene cuatro características, que se presentan en la figura 1. A continuación, se profundizan desde varios estudios los conceptos y las variables que tienen similitud con el caso colombiano. 


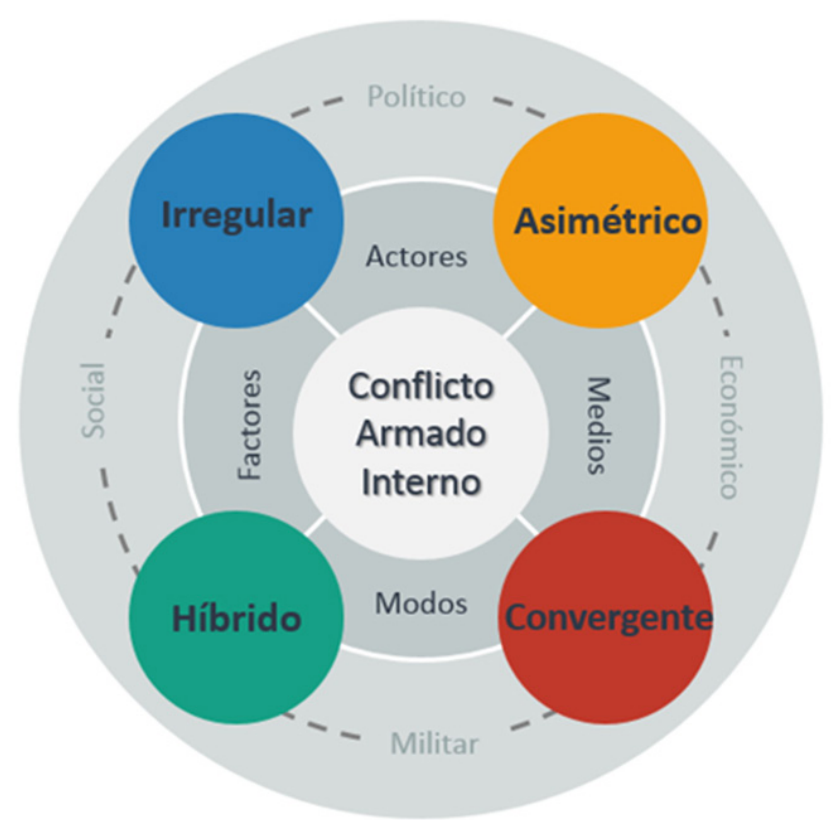

Figura 1. Características del conflicto armado interno en Colombia.

Fuente: Elaborado por los autores con datos de Garay y Ramírez-Benítez (2017).

En primer lugar, de acuerdo con la figura 1, el conflicto armado interno se puede entender como irregular. Esta categoría se define a partir de los actores participantes, es decir, se refiere a que no es una confrontación convencional entre Estados, sino que se desarrolla en el ámbito interno. Específicamente, se entiende que la confrontación armada se da entre un actor legal (fuerzas regulares del Estado o también llamadas Fuerzas Armadas) y un actor ilegal, que por lo general es un grupo armado al margen de la ley. En el caso colombiano se encuentran los grupos insurgentes tradicionales - FARC-EP, el ELN, el EPL, el M-19, entre otros-, así como los paramilitares — las AUC y los más recientes, como las Bandas Criminales (BACRIM) o como se reconocen en la actualidad, Grupos Armados Organizados (GAO) y Grupos Delincuenciales Organizados (GDO $)^{7}$ - (Garay \& RamírezBenítez, 2017, p. 423; Torrijos, s. f., p. 2).

7 Por medio de la Directiva Permanente No. 15 del 22 de abril de 2016 del Ministerio de Defensa Nacional, la cual reemplazó a la Directiva Permanente No. 14 del 2011. En esta directriz se integra una nueva percepción sobre el reconocimiento de los actores armados ilegales en las dos clasificaciones enunciadas (GAO y GDO), teniendo en cuenta que para estos no se hace ningún reconocimiento político. 
En segundo lugar, se entiende como asimétrico ${ }^{8}$, es decir, los actores tienen capacidades diferentes ${ }^{9}$ para desarrollar las acciones propias del enfrentamiento armado. Es aquí donde se presentan elementos como lo inusual e inesperado y donde se involucran métodos no convencionales. Por esta razón es relevante que las Fuerzas Armadas desarrollen capacidades de movilidad, proyección, sostenibilidad, inteligencia, acción conjunta y combinada, entre otras, para enfrentar las amenazas que se presenten en el conflicto (Garay \& Ramírez-Benítez, 2017, p. 423; Instituto de Estudios Geoestratégicos y Asuntos Políticos [IEGAP], 2010).

En tercer lugar, tiene características de un conflicto híbrido, es decir, ha escalado hasta ocupar una posición representativa en otros escenarios, como el político, el económico y/o el social, en el sentido de que no se queda meramente en acciones militares (Garay \& Ramírez-Benítez, 2017, p. 423). Hay que tener en cuenta la relevancia teórica del concepto, el cual fue elaborado por los oficiales estadounidenses James Mattis y Frank G. Hoffman (2005), quienes acuñaron el término hybrid warfare para hacer referencia a la posibilidad de que se desarrolle una serie de amenazas diferentes — convencionales, irregulares, terroristas u otrasen diversos ámbitos, que tendrán como objetivo las vulnerabilidades del Estado y cuyo factor relevante es la simultaneidad al combinar todas las formas de lucha (formas y tácticas).

Finalmente, en cuarto lugar, el conflicto armado interno en Colombia se presenta como convergente, es decir, se involucraron fenómenos ilegales de crimen organizado, terrorismo y delincuencia común. Es así como se da la alianza entre actores que desarrollan alguna de estas actividades, pero convergen en un interés, objetivo o fin mutuo, que en varios casos está orientado a un ámbito económico, sin desconocer los demás. Estos actores realizan acciones ligadas a actividades ilícitas como el narcotráfico, la minería ilegal, acciones terroristas, entre otras (Garay \& Ramírez-Benítez, 2017). De este modo, "la convergencia consiste en la aproximación entre actores del crimen organizado y actores terroristas o políticos" (Ardila, Jiménez \& Bernal, 2016).

Adicionalmente, en Colombia el conflicto armado tuvo características propias, como la presencia diferencial del Estado en el territorio, lo cual generó que se confor-

8 En contraposición se encuentran las guerras o conflictos simétricos. Sobre estos últimos, Geiss (2006) menciona "que tienen lugar entre ejércitos estatales de fuerza militar aproximadamente igual o, al menos con estructuras organizativas comparables" (p. 4).

9 Esta asimetría puede remitirse a antecedentes históricos representados, por ejemplo, por David y Goliat, en donde el contraste entre las partes es evidente. Se refiere entonces a una disparidad entre los medios, modos y otros elementos que marcan una diferencia entre las partes. Finalmente, es importante recalcar que no se trata de una característica nueva (Geiss, 2006). 
maran las llamadas "repúblicas independientes", como expresa Trejos (2013). La capacidad estatal para llegar efectivamente a estos territorios era reducida, teniendo en cuenta que a pesar de que la presencia de la Fuerza Pública era significativa, hacía falta que el entramado institucional del Estado se manifestara en estas zonas, las cuales en su mayoría eran rurales y ubicadas en zonas periféricas. De este modo, dichos territorios fueron ocupados por entidades paralelas como las insurgencias, los paramilitares y los narcotraficantes, entre otros, para establecer sus lineamientos.

Sobre esta situación vale la pena hacer referencia a un concepto acuñado por Romero (2006), citado por Trejos (2013), que denominó "desorden duradero", el cual remite a que el gobierno no logra abordar las causas estructurales ni, por ello, las soluciones de ciertas problemáticas, pero tampoco deja colapsar la entidad estatal en su sistema. Es importante mencionar que el Estado tiene el deber de proteger, para lo cual el gobierno nacional establece una hoja de ruta en el Plan Nacional de Desarrollo para abordar los objetivos principales que permiten garantizar los fines constitucionales y, sobre todo, los aspectos que revisten el interés nacional: la supervivencia, el bienestar económico y los valores — como elementos dados desde el neorrealismo- , así como atender las situaciones que más aquejan a la población colombiana y, en general, al Estado. En este contexto, hay problemas de gran complejidad que involucran multiplicidad de ámbitos y actores, por ejemplo, el crimen transnacional organizado, el terrorismo y el narcotráfico, por mencionar los más evidentes, de manera que es necesario involucrar la cooperación internacional para enfrentar estas amenazas comunes.

\section{Apreciación y desarrollo del conflicto armado desde el nivel estratégico}

La estrategia es entendida como un proceso de planeación que involucra la definición de los fines, medios y modos para la consecución de los objetivos de una organización, que, para este caso, es la consecución de los intereses nacionales materializados en la política de gobierno (Sánchez, 2014). Con esta lógica se estructura el planeamiento nacional, que está compuesto por la Estrategia Nacional, definida por el gobierno; la Estrategia Operativa General o Sectorial, definida por el Ministerio de Defensa, y la Estrategia Operativa, empleada por el Comando General de las Fuerzas Militares y donde participa cada una de las Fuerzas. Como se evidencia, el planeamiento está concatenado y se desprende del nivel superior porque el alto gobierno define los objetivos transitorios y los lineamientos de las políticas públicas del sector defensa. 
Una crítica del proceso de configuración de la estrategia nacional — nivel estratégico- es su falta de continuidad en razón a las transiciones del gobierno nacional. Esto genera la transformación permanente y la redefinición de los medios y modos para cumplir los objetivos planteados por el gobierno, que generalmente varían según la naturaleza de su plan de gobierno.

En este sentido, en el nivel estratégico, y en el marco del desarrollo del conflicto armado colombiano, se evidencia que hay un problema permanente en la forma como se categoriza al enemigo interno - o adversario- - . Esto se debe, en parte, a la fractura del sistema político, que delegó la responsabilidad de conducir las hostilidades en manos de un sistema de alternancia bipartidista (partidos Conservador y Liberal) conocido como el Frente Nacional (1958-1974), cuando en realidad se trata de un asunto esencialmente militar que debía estar alineado a marcos internacionales.

Este evento significativo para el planteamiento de las estrategias contra las amenazas internas emergentes generó en su momento un problema para identificar y definir al enemigo, en un contexto internacional marcado por los conflictos internos y la ideologización de los sistemas políticos entre la derecha capitalista y la izquierda revolucionaria en Latinoamérica. Así, el desarrollo del conflicto armado que inició en los años 60 evidencia que hubo inconvenientes en el enfoque de seguridad y en la alineación jurídica que se debía seguir para cumplir los Derechos Humanos y el DIH. Esta situación se generó debido a la falta de articulación en la forma como se emplearon los medios y las capacidades militares contra los nuevos actores (Leal, 1994).

En este sentido, es necesario traer a colación una crítica del sociólogo Armando Borrero Mansilla (2003), quien señala que una de las principales fallas que ha tenido el Estado en relación con la conducción del conflicto es que ha apreciado e identificado de manera errónea la naturaleza de la contraparte, que ha sido nombrada de diferentes maneras, entre otras: enemigo, adversario, revolucionario o criminal. Esta situación está ligada con uno de los principios del DIH: la humanidad, así como con el respeto a los Derechos Humanos que tengan las partes en el marco de la confrontación armada.

Como se mencionó, esta dificultad en identificar a la contraparte en el conflicto surge inicialmente porque la estrategia nacional no ha sido organizada de forma permanente por el Estado y sus intereses, sino por un programa de gobierno que, durante el Frente Nacional, se interesaba más en garantizar la alternancia del poder y mantener lejos de este a cualquier otro actor. Como consecuencia emergieron nuevas expresiones sociales y distintos actores, algunos de los cuales se configuraron 
como potenciales amenazas al Estado. Si bien es claro que el nuevo enemigo fueron los actores armados ilegales, que representan una amenaza para el orden y la estabilidad del Estado, también se debe reconocer que el contexto nacional fragmentado por la disputa tradicional histórica fue el catalizador de nuevas expresiones ideológicas en la región y en el continente. En cualquier caso, lo cierto es que el Estado requería una respuesta inmediata del conductor político para adecuar su estrategia inmediata al nuevo contexto, en la cual el primer paso debía ser definir al adversario como lo que realmente era, una insurgencia.

En contraposición, durante lo corrido del siglo XX, la imagen sobre el enemigo interno se construyó desde el discurso político de la época, una narrativa que desatendía las realidades sociales, económicas y políticas debido a la desarticulación del sistema político, como se ha insistido.

Por lo anterior, se puede hablar de tres hitos que explican el problema de la organización de la estrategia nacional: (1) la separación de los asuntos políticos y militares fue inadecuada, pues estuvo marcada por una fuerte politización social y de los asuntos internos, lo cual impidió que se elaborara una acertada categorización del enemigo; (2) no se reconoció al enemigo armado interno, sino que fue construido en función del discurso político más que por las razones, intereses y necesidades racionales en materia de seguridad; $y$, (3) los lineamientos permanentes que se definían a manera de ley no se ajustaban a la realidad del contexto, en parte porque no había una definición puntual del enemigo, como tampoco se estableció una noción correspondiente ni proporcional sobre los medios jurídicos internacionales y las capacidades con que contaba el Estado.

Frente al primer punto, la separación de los asuntos políticos y militares, cabe señalar que si bien la consolidación de las grandes democracias liberales se ha construido con el supuesto de que el poder civil tiene una supremacía sobre el poder militar, se debe tener en cuenta que el ámbito militar relacionado con la seguridad y defensa es un campo de las Fuerzas Militares y del monopolio de las armas, donde la política — no lo político- debe ser un asunto delimitado en cuestión de responsabilidades y alcances (De Vergottini, 1982). Esto, por supuesto, no se afirma en la necesaria separación de los asuntos políticos y militares, sino en la separación de "la política” entendida como todos aquellos asuntos relacionados con la ideología de partido. En este sentido, "lo político", entendido como todos los asuntos públicos involucrados en la toma de decisiones que afectan la esfera social colectiva, debe estar en constante coordinación con las altas esferas de gobierno y el poder militar.

En el caso colombiano, después del gobierno de Gustavo Rojas Pinilla (19531957), el poder civil rompió radicalmente su relación con el poder militar a partir 
del discurso del expresidente Alberto Lleras Camargo en el Teatro Patria en 1958, cuando ratificó el manejo del orden público a la Fuerza Pública (Uribe, 2013). Este distanciamiento marcó la construcción de políticas públicas de seguridad interna y externa, así como las transformaciones iniciadas en el siglo XX cuando la Policía Nacional pasó de manera transitoria, y luego permanentemente en 1953, a integrar el Ministerio de Defensa Nacional, en el entonces Ministerio de Guerra (Presidencia de la República, 1953).

Estos aspectos configuraron la arquitectura institucional del sector defensa, caracterizada principalmente porque las agendas de los gobiernos nacionales se encaminaban a la estabilidad y el orden interno, de tal manera que les dieron a las instituciones castrenses un papel fundamental en la estrategia nacional. Esto generó que la organización de la estrategia sectorial de defensa se enfocara en la estabilidad de la seguridad interna y, con ello, se fortalecieran políticas públicas de securitización territorial por parte de la esfera militar.

Evidentemente, la principal misión de las Fuerzas Militares es defender el territorio contra las amenazas internacionales, pero debido a las distintas necesidades el Estado ha tenido que diversificar sus objetivos, lo cual ha traído como efecto positivo la adaptabilidad de la esfera militar y la continua articulación de las relaciones civiles en función de las instituciones democráticas, las libertades y los derechos universales. En consecuencia, se puede afirmar que las dinámicas transformaciones del conflicto interno evidencian la necesidad de replantear los medios y modos de comprender al enemigo y de enfrentarlo (Nieto \& Rey, 2008), manteniendo siempre el respeto por los principios universales y la Constitución nacional.

En este contexto, los conductores políticos carecieron de proyección para definir al enemigo de forma categórica, un actor armado ilegal ya influido por una ideología revolucionaria que empleaba estrategias de guerra asimétrica (Jiménez et al., 2017). Este importante aspecto impidió establecer una estrategia contundente que contrarrestara el escalonamiento del conflicto y, en consecuencia, nuevos actores emergieron como expresiones alternativas alineadas a la violencia (autodefensas, narcotraficantes, organizaciones criminales y demás actores ilegales que se mencionaron con anterioridad).

En medio de esta dinámica evolutiva del enemigo, el papel del Estado como garante de los Derechos Humanos - frente a actores ilegales que atentaban contra la población civil— fue cuestionado por desconocimiento y, en algunos casos, por omisión de la realidad del momento. En estos altibajos los conductores políticos no fueron los únicos que tuvieron un grado de responsabilidad, pues la Fuerza Pública también tuvo dificultades, particularmente las Fuerzas Militares, debido a la falta 
de un marco jurídico alineado con marcos internacionales y a la definición de fines, medios y objetivos contra un enemigo puntual insurgente.

En el marco del Derecho Internacional Público, específicamente en el desarrollo del Derecho Internacional Humanitario, la definición de los actores y su naturaleza determina los mecanismos jurídicos, las instancias de sometimiento y los mecanismos que emplea el Estado en el desarrollo de las hostilidades, debido a que la condición de sujeto del derecho define el grado de derechos y deberes.

Para el Comité Internacional de la Cruz Roja (CICR, 2010), la adopción de los Convenios de Ginebra y sus Protocolos Adicionales son regulaciones que amplían la conducción de las hostilidades en el marco tanto de un Conflicto Armado Internacional como de un Conflicto Armado No Internacional. Al respecto de esta normativa internacional, el punto gira en torno al uso legítimo de la fuerza en relación con las condiciones reales y propias del conflicto en particular, donde se destaca que las acciones militares no deben ser indiscriminadas y deben considerar los objetivos militares legítimos. Estas condiciones aplican tanto para las fuerzas del Estado como para los actores beligerantes.

Ahora bien, esta consideración permite plantear la siguiente cuestión: ¿̨por qué el Estado colombiano no reconoció internacionalmente la amenaza interna de los grupos armados ilegales, caso FARC-EP y ELN como actores beligerantes a pesar de establecer acercamientos para la salida negociada del conflicto con otros actores menos dominantes como el EPL y el M-19, incluso con actores ilegales como las AUC? Parte de esta pregunta se responde en el hecho de que la guerra es un asunto de la política — como lo reconoció Carl von Clausewitz en su momento-. Aunque los asuntos militares tienen gran importancia y reconocimiento en la agenda social, la realidad es que la falta de continuidad de las políticas de seguridad y defensa, así como el cambio de la definición de los medios y modos en la estrategia nacional por parte de la transición de los gobiernos, generan rupturas en la continuidad que han impactado en el nivel operativo.

Asimismo, parte de la respuesta yace en razones jurídicas de fondo, como el tipo de reconocimiento que da el gobierno al estatus del actor ilegal, es decir, del estatus de beligerancia a un actor que a la luz de los intereses de la política no debería ser legitimado. Para Mesa-Mejía (2012), el reconocimiento de la beligerancia a un actor armado parte del hecho de que es un acto político que tiene como efecto jurídico limitado y temporal que sea aplicable el DIH, además de conceder derechos, deberes y responsabilidades como sujeto del derecho internacional. Además, este permite al Estado una "definición puntual del enemigo y del empleo de medios jurídicos internacionales y de las capacidades con que contaba el Estado” (p. 67). 
En el marco del Derecho Internacional Público, el reconocimiento de un grupo armado se tiene que ceñir al Protocolo II de los Convenios de Ginebra de 1949, el cual resalta que el actor debe: (1) ser un grupo armado organizado, (2) contar con una dirección de un mando responsable y mantener control en un territorio, (3) tener la capacidad de sostener operaciones militares (Pacheco, 2012). Y, por último, frente a la definición de los lineamientos permanentes en forma de ley, se debe señalar que en Colombia no ha habido una conceptualización jurídica específica del actor ilegal armado que se ajuste a la realidad del contexto social ni al derecho internacional en materia del DIH. Así las cosas, la falta de esta definición concreta ha generado una debilidad en la determinación de los medios jurídicos internacionales conformes a las capacidades con las que cuenta el Estado para el sometimiento de los actores, un aspecto que se ha corregido en parte con la puesta en marcha del Derecho Operacional, pero que no soluciona el problema de fondo.

Ahora bien, ¿por qué es necesario establecer un marco normativo claro que defina al enemigo interno? Durante las últimas dos décadas, la concepción sobre cómo se deben enfrentar las amenazas armadas ha dependido del jefe de gobierno de turno, lo cual ha generado una ruptura periódica de las estrategias nacionales cada vez que existe un cambio de gobierno (Ardila et al., 2018). En este sentido, la seguridad y defensa nacionales, un ámbito que necesariamente debe ser gestionado por la Fuerza Pública en coordinación con el poder civil y sus diferentes instancias e instituciones, ha carecido de respaldo político para que sea definido como un interés de Estado.

Por lo anterior, el Estado colombiano debe cambiar la concepción que tiene para redefinir sus objetivos en el campo de la seguridad y defensa nacional. Es fundamental que establezca objetivos puntuales permanentes, es decir, que no se transformen con los cambios de gobierno ni frenen los avances tras cada elección, de manera que la ley de seguridad y defensa permita a largo y mediano plazo organizar la estrategia nacional.

\section{Percepción actual del conflicto armado en el periodo del posacuerdo}

Como se ha mencionado, para determinar que existe un conflicto armado interno se deben evidenciar dos criterios principales (CICR, 2016): en primer lugar, las partes en conflicto deben estar organizadas, es decir, deben tener una cadena de mando, una estructura específica, capacidad de entrenamiento, de mantener 
operaciones militares durante un tiempo prolongado y, sobre todo, capacidad de administrar/controlar un territorio. En segunda medida, se debe considerar que la intensidad de las hostilidades, la frecuencia y duración de los enfrentamientos, los medios utilizados —entiéndase el armamento y el equipamiento-, la cantidad de personas heridas y muertas en el marco de las hostilidades, y, en particular, en relación con la población protegida y no combatiente, pueden generar algún tipo de implicación internacional. En este sentido, la definición de la estrategia, los criterios de definición de los actores y del conflicto son aspectos importantes que se deben tener en cuenta en las políticas públicas, buscando, en lo posible, la comunidad de estas como un interés nacional imperativo.

Estos criterios del CICR (2016) permiten analizar el caso colombiano para categorizar y reconocer el conflicto armado interno en el país. Adicionalmente, como se mencionó, en el año 1996 entró en vigor la aplicación del Protocolo II Adicional de los Convenios de Ginebra, toda vez que el Estado colombiano lo había firmado y ratificado junto con los Convenios generales. En este escenario hay que tener en cuenta que cada ente estatal tiene autonomía, soberanía y comprende unos factores situacionales diferenciales para aplicar dicho Convenio, ya que en términos generales no existe un procedimiento vinculante que obligue y establezca de qué manera se debe cumplir. Sin embargo, es evidente que, al ratificar esta normatividad, el Estado se ve comprometido en acoger y aplicar las normas (CICR, 2016).

Una vez se ha hecho claridad sobre el referente conceptual, a continuación se retoma la perspectiva del jurista Rodrigo Uprimny Yepes (2005), quien afirma que es relevante definir la situación de conflicto que vive Colombia porque esto trae implicaciones jurídicas y políticas. Al respecto, enfatiza que "parece claro que no pueden ser iguales las estrategias jurídicas o políticas para enfrentar una amenaza terrorista que para superar un conflicto armado" (párr. 2). Además, de esta manera se clarifican los medios y los modos para desarrollar los lineamientos que contrarrestan las acciones y situaciones negativas y de violencia que se generan en el país.

En este sentido, desde el año 2002, los lineamientos del entonces presidente Álvaro Uribe Vélez estaban orientados a reconocer una "amenaza terrorista". Como expone Olave (2012), en el primer periodo presidencial se refería a estos grupos ilegales como terroristas y, en el segundo, como narco-terroristas, "luego de que perdieran su estatus de beligerancia al término de los diálogos de paz fallidos con el expresidente Andrés Pastrana Arango (1998-2002)”.

A partir del año 2011 se generó una ruptura con la percepción del mandato anterior, ya que el gobierno de Juan Manuel Santos reconoció el conflicto armado 
interno por medio de la Ley de Víctimas y Restitución de Tierras (Ley 1448, 2011), en la cual se enuncian tácitamente los derechos y medidas de protección de "las víctimas del conflicto armado interno”, además de las medidas para la atención, la asistencia y la reparación integral. Textualmente, el objeto de esta ley indica que "se dictan medidas de atención, asistencia y reparación integral a las víctimas del conflicto armado interno y se dictan otras disposiciones". Además, en el artículo tercero del título primero de esta ley se menciona:

Se consideran víctimas, para los efectos de esta Ley, aquellas personas que individual o colectivamente hayan sufrido un dańo por hechos ocurridos a partir del $1 .^{\circ}$ de enero de 1985, como consecuencia de infracciones al Derecho Internacional Humanitario o de violaciones graves y manifiestas a las normas internacionales de Derechos Humanos, ocurridas con ocasión del conflicto armado interno.

Además de esto, en el articulado de la ley se enuncian causas y efectos en el marco del conflicto armado, por cuanto constituye una normativa del ordenamiento constitucional que clarificó la distinción específica respecto a la confrontación entre las Fuerzas Militares y los grupos alzados en armas, más conocidos como grupos armados ilegales. Por supuesto, como era de esperarse, la discusión de reconocer o no el conflicto armado interno en la etapa previa y durante el trámite de la ley causó gran polarización de los sectores políticos.

Asimismo, es importante considerar la perspectiva de los organismos externos respecto al reconocimiento del conflicto. Como se ha visto a lo largo del escrito, el CICR (2019) ha sido un organismo relevante que ha acompañado el desarrollo del conflicto armado en Colombia, ya que ha estado en el país desde 1969, cuando tuvo su primer acercamiento con una actividad que realizó en las cárceles colombianas con el fin de promover mejores condiciones para los reclusos en el periodo de detención. Desde entonces, y en el marco del conflicto armado interno, el CICR ha realizado acciones de atención a víctimas en los diversos hechos desafortunados que han acontecido en la realidad colombiana, por ejemplo, en El Salado (2000), en Mapiripán (1997), entre otros.

También se debe destacar que esta organización ha tenido diálogo e interacción sistemáticos con las Fuerzas Armadas colombianas. Además, a partir de 1994 empezó a establecer contacto con grupos armados organizados en Colombia, lo cual ha sido un medio para difundir la normatividad humanitaria y acompañar su adopción y aplicación.

Actualmente, el CICR (2018) ha realizado diversos pronunciamientos alrededor del reconocimiento y caracterización del conflicto armado. Por medio de su 
delegado, Christoph Harnisch, ha señalado que en su consideración existen cinco tipos de conflicto armado en Colombia, además de enfatizar la perdurabilidad que ha tenido la violencia y la complejidad del contexto. El diplomático mencionó que la firma del Acuerdo de Paz es un avance, pero advirtió que su garantía es que se consolide durante un largo periodo.

De igual forma, Harnisch recalcó que la categoría de posconflicto no puede reducir el impacto del conflicto, pues si bien se realizó un diálogo con uno de los actores —las FARC-EP_, aún se manifiestan varios más en el panorama de la violencia en Colombia. Por esta razón, se especifica que el posacuerdo es una etapa posterior a la firma de los acuerdos en la que se desarrollan las acciones de implementación y verificación (Oquendo, 2019). Volviendo al primer punto, se tiene que los cinco tipos de conflicto armado que tiene Colombia según el CICR (2018) son:

1. Ejército de Liberación Nacional: insurgencia que tiene presencia principalmente en zonas como Chocó, Nariño, Cauca y Catatumbo. Con este grupo armado el Estado colombiano ha mantenido su intención de diálogo, pero interrumpió el proceso por las acciones de violencia que continúa realizando.

2. Clan del Golfo: mantiene presencia en el territorio y afecta la estabilidad de la región y a la población.

3. Ejército Popular de Liberación: estructura armada ilegal que se encontraba debilitada en el año 2016, pero que logró fortalecerse en el 2017.

4. Disidencias de las FARC-EP: grupos residuales que se apartaron del Proceso de Paz. El CICR identifica al Frente 1, 7 y 40.

5. Los enfrentamientos entre el ELN y el EPL forman el quinto conflicto (figura 2).

Esta categorización del conflicto armado colombiano que hace el CICR tiene varias consideraciones para el análisis. En primer lugar, se debe mencionar que a diferencia de la forma como generalmente se ha entendido este fenómeno, es decir, como un solo conjunto y un solo periodo histórico integrado por actores, causas, situaciones específicas, entre otros aspectos, el organismo plantea esta tipología desde los actores armados ilegales que se encuentran inmersos en el conflicto. Es decir, la interpretación del CICR de particularizar el conflicto dependiendo del tipo de actor ilegal que se enfrenta al Estado colombiano constituye una nueva manera de enfocar el conflicto. No obstante su novedad, la pertinencia de esta clasificación ha sido cuestionada. 


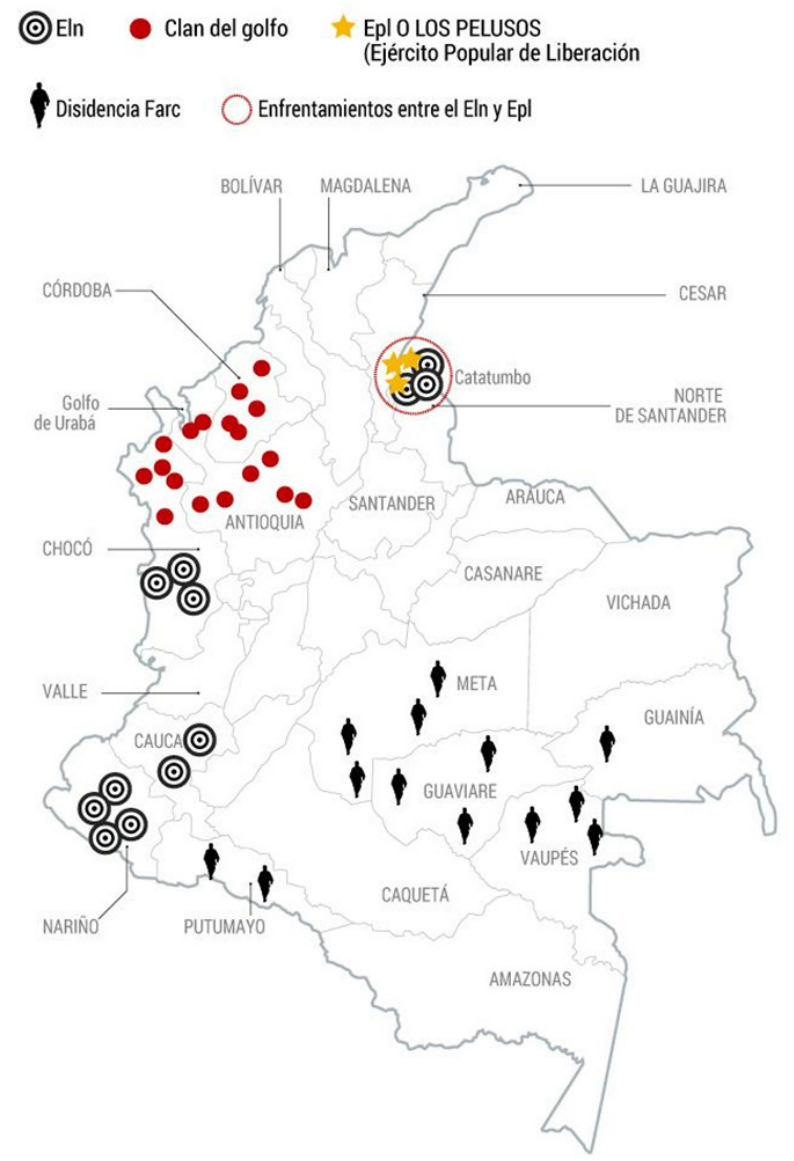

Figura 2. Los cinco conflictos armados en Colombia según Christoph Harnisch, jefe de la delegación del CICR en Colombia.

Fuente: Castrillón (2019).

En este sentido, pese a las declaraciones del organismo internacional, hay que enfatizar en los principios de soberanía nacional y autonomía que tiene el Estado, ya que entender y particularizar el conflicto dependiendo del actor armado ilegal abre nuevas cuestiones relacionadas con los modos y medios para enfrentarlos. Asimismo, hay que recalcar que el Estado busca garantizar y preservar los lineamientos constitucionales consagrados en el artículo tercero, en donde se estipulan los fines del Estado colombiano. De este modo, hay que tener claro que la articulación de la estrategia nacional en sus variables de medios y modos es direccionada desde el gobierno nacional como el conductor político de los lineamientos para cumplir los objetivos propuestos orientados a la supervivencia del Estado. 
Además, en su calidad de vocero del CICR en Colombia, Harnisch también ha señalado que en Colombia existe un conflicto armado no internacional, para lo cual se basa en la adopción del Protocolo II Adicional en 1977, en donde el Estado colombiano adquirió compromisos en materia humanitaria desde la perspectiva del DIH. A parte de esto, trae a colación la experiencia que ha tenido el CICR en el territorio colombiano y cómo esta evidencia que se trata de este tipo de conflicto, a partir del cual se determina el modo en que se debe proteger a la población civil (Castrillón, 2019).

Finalmente, cabe señalar que con el proceso de paz entre el Estado colombiano y las FARC-EP, los grupos armados organizados reconfiguraron sus estructuras en el territorio. Por ejemplo, las insurgencias como grupos residuales de esta guerrilla —conocidas también como disidencias—, el ELN, el EPL y otros grupos derivados como las GAO y las GDO. Adicionalmente, se han desencadenado diversas confrontaciones no solo en contra del Estado, principalmente contra la Fuerza Pública, sino también entre estos (CICR, 2018).

En este sentido, en el posacuerdo los objetivos establecidos son implementar lo pactado ${ }^{10}$ y el cese de las acciones de violencia en medio de un conflicto armado que aún se desarrolla en el territorio. Entendiendo que para lograrlos y

para que el proceso de construcción de paz sea efectivo, la transición del conflicto hacia el periodo de estabilidad debe darse por medio de elementos fundamentales como la verdad, la justicia, la reparación y la no repetición, que se articulan en el mecanismo propio establecido para este tipo de procesos, como lo es la Jurisdicción Especial para la Paz, necesaria para que pueda darse una reconciliación nacional en el largo plazo. (Caldera, Cubides-Cárdenas, Ardila-Castro \& Ramírez-Benítez, 2019, p. 188)

10 Para ello se debe tener en cuenta la necesidad de : “(i) enfocarse en las zonas rurales-vulnerables atendiendo las necesidades básicas y contrarrestando los efectos derivados a la pobreza; (ii) la importancia de la reconstrucción de lo social, teniendo el ámbito local como el escenario más próximo; (iii) la presencia de las instituciones del Estado en todo el territorio nacional es una necesidad inmediata, con el fin de solventar las necesidades, vacíos y carencias que han tenido varias poblaciones en diversas zonas del país" (Cubides-Cárdenas, Ramírez-Benítez \& Betancourt, 2018, p. 226). 


\section{Conclusiones}

Se puede concluir que el Estado ha evidenciado históricamente la evolución sistemática de las amenazas tradicionales, conformadas, por ejemplo, por las guerrillas revolucionarias y otras que han emergido derivadas del conflicto y relacionadas con las organizaciones criminales. Estos aspectos generaron un problema en la forma como se categorizaba las amenazas internas y, por ende, una definición puntual de la tipología de conflicto, lo cual impidió que se estableciera una estrategia nacional contundente para confrontar a los actores armados ilegales. En este sentido, las Fuerzas Militares y la Policía Nacional han tenido que cambiar sus funciones naturales de acuerdo con las necesidades para contrarrestar las dinámicas del conflicto, un punto que debe ser considerado como un interés nacional.

A este aspecto se suma la falta de una ley de seguridad y defensa permanente que, además de reconocer el tipo de conflicto, estableciera un marco jurídico permanente y no transitorio alineado con los principios internacionales de respeto a los Derechos Humanos y al DIH, de manera que le permitiera al Estado cumplir el objetivo de definir los fines, medios y modos para confrontar las amenazas o desafíos. Si bien las fuerzas han avanzado en el cumplimiento de las normativas nacionales e internacionales, los miembros de la Fuerza Pública carecen de seguridad jurídica debido al cambio de contexto y a la convergencia de los múltiples actores ilegales.

En los últimos diez años el Estado colombiano y, en especial, su Fuerzas Militares se han destacado internacionalmente por respetar y cumplir su misión constitucional en el marco de un conflicto complejo que no corresponde a su misión natural. Este aspecto ha generado que las Fuerzas desarrollen funciones de apoyo a la seguridad interna de la nación, por eso se evidencia su compromiso con el respeto de los Derechos Humanos al incorporar el Derecho Operacional.

En consecuencia, y teniendo en cuenta la necesidad de definir una ley de seguridad y defensa, se deben establecer esfuerzos conjuntos para que la política de seguridad y defensa mantenga una estrategia permanente que no dependa de los cambios de gobierno, de tal manera que haya continuidad operativa sin que se determine o condicione por un discurso. Por lo tanto, y teniendo en cuenta el escenario de posacuerdo, se propone que el Estado colombiano genere un marco normativo que (1) defina el tipo de conflicto específico, (2) categorice de manera puntual los actores ilegales involucrados y (3) defina las jurisdicciones y funciones de las Fuerzas, que deben orientar sus acciones a estabilizar los territorios y desarticular las estructuras criminales, teniendo en cuenta que existen particularidades en 
cada región que deben ser consideradas en favor de las normativas internacionales, como se ha desarrollado hasta el momento, pero más aún, no se debe permitir la prolongación del conflicto armado.

\section{Conflicto de intereses}

Los autores declaran que no existe ningún potencial conflicto de interés relacionado con este capítulo.

\section{Financiación}

Los autores no declaran fuente de financiamiento para la realización de este artículo.

\section{Referencias}

Ardila, C., Jiménez, J., \& Acosta, H. (2018). Una aproximación a la Política de Seguridad y Defensa desde la cultura de seguridad y defensa nacionales. En G. Eljach Pacheco, J. A. Escobar Solano, L. M. Meneses, \& G. F. Niño Contreras (Comps.), Políticas públicas y gestión pública en Colombia: estudios de caso (pp. 134-149). Recuperado de http://cael.senado.gov.co/cael/publicaciones/69-serie-11/file

Barcelona Centre for International Affairs [CIDOB]. (s. f.). Conflicto en Colombia: antecedentes históricos y actores [información en página web]. Recuperado de https://www.cidob.org/ es/publicaciones/documentacion/dossiers/dossier_proceso_de_paz_en_colombia/dossier_ proceso_de_paz_en_colombia/conflicto_en_colombia_antecedentes_historicos_y_actores

Borrero Mansilla, A. (2003). La actualidad del pensamiento de Carl von Clausewitz. Revista Estudios Sociales, 16, 23-28. https://revistas.uniandes.edu.co/doi/pdf/10.7440/res16.2003.02

Caldera Ynfante, J., Cubides-Cárdenas, J., Ardila-Castro, C., \& Ramírez-Benítez, E. (2019). El rol del militar en el posconflicto en Colombia para la construcción de paz. Opción, 35(25), 182-235. https://produccioncientificaluz.org/index.php/opcion/article/view/32310/33724

Cárdenas, J. D. (2013). Opinión pública y proceso de paz: actitudes e imaginarios de los bogotanos frente al proceso de paz de La Habana entre el gobierno colombiano y la guerrilla de las FARC. Revista Ciudad Paz-ando, 6(1), 41-58.

Castrillón, G. (10 de febrero de 2019). "En Colombia sí hay un conflicto armado no internacional": CICR. El Espectador. Recuperado de https://www.elespectador.com/colombia2020/pais/en-colombia-si-hay-un-conflicto-armado-no-internacional-cicr-articulo-857629

Comité Internacional de la Cruz Roja [CICR]. (1977). Protocolo II Adicional a los Convenios de Ginebra de 1949 relativo a la protección de las víctimas de los conflictos armados sin carácter internacional [información en línea]. Recuperado de https://goo.gl/knfzbG

Comité Internacional de la Cruz Roja [CICR]. (2004). ¿Qué es el Derecho Internacional Humanitario? [documento en línea]. Servicio de asesoramiento en Derecho Internacional Humanitario. Recuperado de https://goo.gl/dScSxM 
Comité Internacional de la Cruz Roja [CICR]. (29 de octubre de 2010). El DIH en la conducción de hostilidades [información en línea]. Recuperado de https://www.icrc.org/es/doc/war-and-law/ conduct-hostilities/overview-conduct-of-hostilities.htm

Comité Internacional de la Cruz Roja [CICR]. (5 de febrero de 2016). Colombia: 10 preguntas para entender el Protocolo II de los Convenios de Ginebra [información en página web]. Recuperado de https://www.icrc.org/es/document/colombia-10-preguntas-para-entender-el-protocolo-ii-de-los-convenios-de-ginebra

Comité Internacional de la Cruz Roja [CICR]. (6 de diciembre de 2018). Cinco conflictos armados en Colombia ¿qué está pasando? [información en página web]. Recuperado de https://www.icrc. org/es/document/cinco-conflictos-armados-en-colombia-que-esta-pasando

Comité Internacional de la Cruz Roja [CICR]. (12 de marzo de 2019). Medio siglo del CICR en Colombia [información en página web]. Recuperado de https:/www.icrc.org/es/document/ medio-siglo-en-colombia

Corbacho, A. L. (2011). Evolución del pensamiento estratégico en las relaciones internacionales. Serie Documentos de Trabajo 477 [Universidad del Cema]. Recuperado de https://ucema.edu.ar/ publicaciones/download/documentos/477.pdf

Cubides-Cárdenas, J., Ramírez-Benítez, E., \& Betancourt, L. (2018). Sujetos internacionales y víctimas: actores fundamentales para la reconciliación y promoción de Derechos Humanos en Colombia en el posconflicto. En M. Torres (Ed.), Diálogos: los Derechos Humanos después del Acuerdo. Bogotá, D. C.: Ediciones Escuela Superior de Guerra. https://doi.org/10.25062/9789585698352.06

De Vergottini, G. (1982). La supremacía del poder civil sobre el poder militar en las primeras constituciones liberales europeas. Revista de Derecho Constitucional, 2(6), 9-34.

Instituto de Estudios Geoestratégicos y Asuntos Políticos [IEGAP]. (2010). Evaluación de la Politica de Defensa y Seguridad Democrática, 2002-2010. Bogotá, D. C.: Universidad Militar Nueva Granada. Recuperado de https://goo.gl/izqPGX

Garay Acevedo, C., \& Ramírez Benítez, E. (2017). Los factores estratégicos de Colombia en seguridad y su posicionamiento regional en el posconflicto. En J. Cubides-Cárdenas, \& J. Jiménez Reina (Eds.), Desafios a la seguridad y defensa nacional de Colombia: teoría y praxis (pp. 456-458). Bogotá, D. C.: Escuela Superior de Guerra. https://doi.org/10.25062/9789585625259

Geiss, R. (2006). Las estructuras de los conflictos asimétricos. International Review of the Red Cross, 864. https://www.icrc.org/es/doc/assets/files/other/irrc_864_geiss.pdf

Jiménez R., Acosta, H., \& Múnera, A. (2017). Las disidencias de las FARC: estructuras criminales configuradas como Grupos Armados Organizados - GAO_. En J. Cubides-Cárdenas, \& J. Jiménez, J. (Eds.), Desafios para la seguridady defensa nacional de Colombia: teoria y praxis (pp. 351-406). Bogotá, D. C.: Escuela Superior de Guerra. https://doi.org/10.25062/9789585625259

Leal, F. (1994). El oficio de la guerra. La seguridad nacional en Colombia. Bogotá, D. C.: Tercer Mundo. Mattis, J., \& Hoffman, F. (2005). Future warfare: the rise of hybrid warfare. U.S. Naval Institute Proceedings, 132(11), 30-32.

Mesa-Mejia, J. (2012). La práctica del reconocimiento de beligerancia y la distinción entre conflictos armados internos e internacionales. Dos temas polémicos en el Derecho Internacional Humanitario. Cuaderno de Ciencias Políticas IV. Diálogo de Saberes, 64-70. 
Nieto, R., \& Rey, P. (2008). Las autodefensas y el paramilitarismo en Colombia. Confines de Relaciones Internacionales y Ciencia Politica, 4(7), 43-52.

Olave, G. (2012). La construcción del conflicto armado en el discurso del presidente Juan Manuel Santos. Análisis Político, 25(76), 159-174.

Oquendo, C. (21 de julio de 2019). Hay cinco conflictos armados hoy en Colombia. El Pais. Recuperado de https://elpais.com/internacional/2019/07/20/colombia/1563649226_997490. html

Pacheco, R. (2012). Reconocimiento de beligerancia [documento en página web]. Recuperado de http://www.unilibrebaq.edu.co/unilibrebaq/pdhulbq/publicaciones/reconocimientobeligerancias.pdf.

Presidencia de la República. (16 de julio de 1953). Por el cual se incorpora a las Fuerzas Armadas el Cuerpo de Policía Nacional. Diario Oficial, 28248.

Rehm, L. (2014). La construcción de las subculturas políticas en Colombia: los partidos tradicionales como antípodas políticas durante La Violencia, 1946-1964. Hist. Soc., 27, 17-48. https://doi. org/10.15446/hys.n27.44582

República de Colombia. (10 de junio de 2011). Ley de Víctimas y Restitución de Tierras [Ley 1448 de 2011]. Congreso de Colombia. Diario Oficial, 48096. Recuperado de http://www.suin-juriscol. gov.co/viewDocument.asp?ruta=Leyes $/ 1680697$

Torrijos, V. (s. f.). Cartografía del conflicto: pautas interpretativas sobre la evolución del conflicto irregular colombiano [información en página web]. Recuperado de https://goo.gl/zejyXL

Trejos, L. F. (2013). Dominio territorial y control social en el conflicto armado colombiano. En C. Barreira, J. Tavares Dos Santos, J. Zuluaga, R. González, \& F. González (Coords.), Conflictos sociales, luchas sociales y politicas de seguridad ciudadana. México, D. F.: Universidad Autónoma del Estado de México, Instituto Latinoamericano de Estudios Avanzados \& Consejo Latinoamericano de Ciencias Sociales.

Uprimny Yepes, R. (2005). ¿Existe o no conflicto armado en Colombia? [documento en línea]. Recuperado de https://www.dejusticia.org/existe-o-no-conflicto-armado-en-colombia/

Uribe, S. (2013). Las relaciones político-militares en Colombia y su efecto sobre el rol de las FF.MM. [información en página web]. Recuperado de https://laparada.uniandes.edu.co/index.php/ la-revista/la-revista-4/los-tesos/las-relaciones-politico-militares-en-colombia-y-su-efecto-sobre-el-rol-de-las-ff-mm

Vallejo Rubiano, H., Ramírez-Benítez, E., \& Cubides-Cárdenas, J. (2017). Corpus Iuris en materia de víctimas del conflicto armado interno en Colombia. En H. Vallejo Rubiano, \& J. CubidesCárdenas (Eds.), El conflicto armado interno en Colombia: análisis jurídico de protección a las victimas y del medio ambiente (pp. 51-86). Bogotá, D. C.: Ediciones Escuela Superior de Guerra. Recuperado de https://esdeguelibros.edu.co/index.php/editorial/catalog/view/22/19/160-1 